\title{
Cell Loss Ratio of ATM Multiplexer Controlled by Leaky Bucket Type UPC
}

\author{
Kei YAMASHITA and Youichi SATO \\ NTT Optical Network Systems Laboratories \\ 1-2356 Take, Yokosuka, Kanagawa, 238-03 Japan \\ Tel: +81468592015 Fax: +81468598569 \\ Email:kei@ntttsd.ntt.jpyouichi@ntttsd.ntt.jp
}

\begin{abstract}
For ATM multiplexers controlled by LB (Leaky Bucket) type UPC (Usage Parameter Control), the relation between Cell Loss Ratio (CLR) and the periodic pattern of cell arrival is investigated. First, simulations investigate in detail how the CLR depends on the periodic pattern and it is shown that the conventional "worst" pattern of periodic cell arrival is not always the worst. Thus a bandwidth allocation method based on the "worst" pattern may not guarantee the QOS (Quality of Service). Second, it is examined how the CLR-periodic pattern relation is modified by the LB algorithm parameters. To this end, we approximate the survivor function value of the queue length. The approximation is based on Benes's G/G/1 result and a "shifted normal approximation". It is shown that the sets of LBA parameters for which the conventional "worst" pattern is not the worst are rather limited.
\end{abstract}

Keywords

ATM, Bandwidth Allocation, Leaky Bucket, Cell Loss Ratio, Worst Case Traffic

\section{INTRODUCTION}

When operating an ATM network, the network operator should guarantee to every connection the desired Quality of Service (QOS), which may be defined by the Cell Loss Ratio (CLR), Cell Transfer Delay and so on. At the same time, the network operator should not waste network resources but use them as efficiently as possible. The set of actions taken by the network to achieve the above objectives can be aggregated to form the traffic control function (ITU-T, 1993).

The two most important functions in the traffic control are Connection Admission Control (CAC) and Usage Parameter Control (UPC). CAC judges whether a requested connection may be established given its requested QOS while maintaining the QOS guaranteed to all existing connections. CAC needs a Bandwidth Allocation (BWA) method, i.e., an algorithm to calculate the bandwidth needed to set up any connection. UPC monitors and controls users cell streams at User Network Interfaces (UNI). If the cell stream of a user's connection violates its contract, UPC should detect this and discard or tag cells of the connection. Thus the UPC method determines the characteristics of cell streams entering a network; accordingly, 
the BWA method depends upon the UPC method specified.

Monitoring of cell streams by users themselves is called conformance testing. The algorithm of conformance testing is likely to be standardized internationally on the Leaky Bucket Algorithm (ATM forum, 1993) (ITU-T, 1993). When user's conformance testing is performed by the Leaky Bucket Algorithm (LBA), an accurate UPC is created by also adopting the LBA.

A BWA method that can strictly guarantee any QOS and maximize the utilization of transmission links has not been established yet for LBA-based UPC. In the present paper, we investigate multiplexing characteristics of cell streams conforming to $\mathrm{LBA}$ in order to attack the ideal BWA method.

First, we perform computer simulations on the multiplexing of periodic cell streams permitted by the LBA. We investigate in detail by simulation how the CLR changes when the periodic pattern changes. Second, we examine how the relation between the CLR and the periodic pattern depends upon source LBA parameters.

Section 2 describes the system model. Section 3 shows the simulation results. Section 4 proposes an approximation for the survivor function (s.f.) values of the queue length. Using this approximation, we calculate the CLRs of many kinds of periodic patterns for many sets of LBA parameters. Section 5 argues the impact of our results on BWA. The paper is concluded in section 6 .

\section{MODEL FOR MULTIPLEXING ATM CONNECTIONS}

We model the multiplexing of ATM connections as follows. Figure 1 shows the typical network configuration. Each connection (Virtual Path (Sato, K., 1990) or Virtual Circuit) is multiplexed at a network node (switch, XC and etc.) after passing through a LBA type UPC. If the cell slot of the transmission link is not available when a cell arrives at a node, the cell waits in a buffer for an empty slot. If the buffer is occupied, the next arriving cell is discarded. The First-In-First-Out (FIFO) discipline is applied.

UPC is performed by two LBAs with different parameters (Niestegge (1990), dual leaky bucket). One LBA is for the peak rate of cell arrival and the other is for the average rate (sustainable cell rate in ATM forum (1992)). We define LBA as the one described by Niestegge (1990). The UPC uses a counter which is incremented by 1 when a cell arrives. While no cell arrives, the value of the counter is decremented at the average arrival rate. When the counter reaches its agreed maximum value, all incoming cells are rejected. We call the maximum value of the counter "the depth of the LB" hereafter. The above LBA definition is equivalent with the one given in annex 1 of ITU-T (1993), though there are some differences with respect to the definitions of parameters. For simplicity, we fix the depth of the peak rate LB to 1 throughout this paper, which means no CDV is considered.

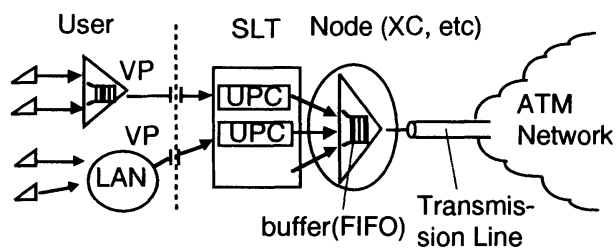

Figure 1 Multiplexing of Virtual Paths. 
(a) Basic pattern (Conventional worst pattern, greedy on-off pattern (Worster, 1994)) maximum burst

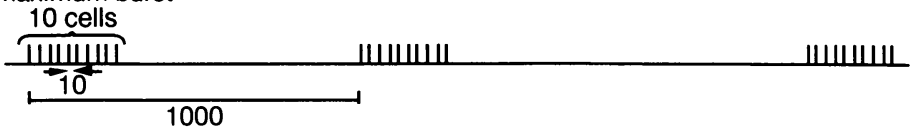

(b) Maximum Burst +10 cells arriving at the average rate (YSS pattern (Worster, 1994))

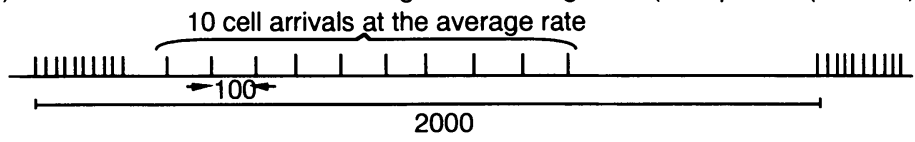

(c) Maximum Burst +4 cells arriving at the average rate

4 cell arrivals at the average rate

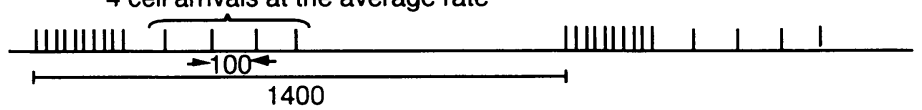

Figure 2 The conventional "worst" pattern of periodic cell arrival and two other patterns that pass through the same LBA.

\section{SIMULATION OF MULTIPLEXING OF CELL STREAMS}

\section{1 The worst pattern}

A network has to make the CLR of each connection less than the permissible maximum value negotiated beforehand. Since the only constraint on the user cell stream is that it should conform to the LBA, the network must prepare for the worst case traffic permitted by the UPC (ITU-T, 1993).

It is commonly believed that there is a periodic arrival pattern called "the worst pattern" that causes the largest CLR among all periodic patterns. Phases of arrival patterns of multiplexed connections are assumed to be random, i.e., subject to uniform distribution independently. The worst pattern was generally believed to be a simple on-off pattern like Figure 2 (a) (Kositpaiboon, 1990), which we call the basic pattern, following Yamanaka et al.(1992). In the basic pattern, cell arrival at the peak rate continues as long as allowed (which we call the maximum burst), and thereafter no cell arrives.

It has not been proved, however, that the basic pattern is the worst pattern. Thus we simulate the multiplexing of periodic cell streams in order to compare the CLRs of the basic pattern to those of other patterns.

\section{2 Review of the results of Yamanaka et. al and Worster}

Yamanaka et al. (1992) found several patterns that caused larger "survivor function (s.f.) values" of the queue length than the basic pattern. One of them is shown in Figure 2 (b). Figure 2 (a) is the basic pattern for the same LBA parameters. In pattern (b), 10 cells arrive at the average rate after the maximum burst. The LBA parameters are as follows: the depth of the LB, the peak arrival rate, and the average arrival rate are 9.1,0.1, and 0.01, respectively. (Arrival rates are normalized by the transmission rate of the link.) Thus the number of cells in the maximum burst is 10 . The number of multiplexed cell streams is 99 . 
Table 1 CLRs and Survivor Function Values for the 150 cell buffer

\begin{tabular}{lccc}
\hline & Fig.2 (a) & Fig.2 (b) & Fig.2 (c) \\
\hline Cell Loss Ratio & $2.0220 \times 10^{-4}$ & $2.1130 \times 10^{-4}$ & $5.2293 \times 10^{-4}$ \\
& $\pm 1.47 \times 10^{-6}$ & $\pm 1.00 \times 10^{-6}$ & $\pm 2.27 \times 10^{-6}$ \\
Survivor func. value & $1.4527 \times 10^{-3}$ & $2.5269 \times 10^{-3}$ & $4.7341 \times 10^{-3}$ \\
& $\pm 1.02 \times 10^{-5}$ & $\pm 1.25 \times 10^{-5}$ & $\pm 2.08 \times 10^{-5}$ \\
& & \\
\# Survivor function value: Pr[queue>150] & & \\
\# 95\% confidence interval & & \\
\hline
\end{tabular}

Table 2 CLRs for the 150 cell buffer from Worster (1994)

\begin{tabular}{lll}
\hline & Fig.2 (a) & Fig.2 $(b)$ \\
\hline Cell Loss Ratio & $\begin{array}{l}2.97 \times 10^{-4} \\
\pm 0.34 \times 10^{-4}\end{array}$ & $\begin{array}{l}2.79 \times 10^{-4} \\
\pm 0.25 \times 10^{-4}\end{array}$ \\
\# 95\% confidence interval & & \\
\hline
\end{tabular}

From this result, Yamanaka et al. concluded that the basic pattern is not always the worst pattern. Examples of s.f. values are shown in Table 1. We obtained these through simulations.

Worster (1994) pointed out that Yamanaka's conclusion implicitly uses this assumption:

Assumption 1 (assumption B in Worster (1994))

"an arrival process that produces longer queues in an infinite queue will produce higher loss in a finite queue."

Worster considered that this assumption is a result of the stochastic queuing theory and found that it can not always be applied to the deterministic queuing case that we are now discussing. He performed a simulation that showed pattern (b) (called as "YSS pattern" in Worster (1994)) does not yield larger "CLR" than the basic pattern (a) (called the "greedy onoff pattern in Worster (1994)), though pattern (b) yields larger "s.f. values" than the basic pattern. He concluded that assumption 1 is not always true and Yamanaka's result is not enough to deny the statement that "the basic pattern is the worst pattern".

Worster, however, investigated only one pattern, pattern (b), for the LBA parameters Yamanaka used, though there are an infinite number of periodic patterns that pass through the LBA with identical parameters. (Worster performed another simulation for another set of LBA parameters, and he obtain the same conclusion.) Thus the conjecture that "the basic pattern is the worst pattern" still lack of a firm proof, as Worster also mentioned. 


\section{3 Re-simulation of the Yamanaka-Worster case}

We investigated patterns other than (a) and (b) in Figure 2 for the same LBA parameters used by Yamanaka and Worster. We found that some patterns yield larger "CLR" than the basic pattern.

Table 1 shows the CLR and the s.f. values obtained by our simulation for patterns (a), (b) and (c) in Figure 2. Table 2 is the result quoted from Worster's paper. We reconfirm that the CLR of pattern (b) is almost the same as that of pattern (a), though the actual values are slightly different in Table 1 and Table 2. (Some differences of simulation models or pseudo-random number generators used may cause this discrepancy, but the origin is not clear. See Appendix A and B.) So assumption 1 does not seem always true (we'll revisit this problem in section 3.4). The CLR of pattern (c), however, is more than double that of

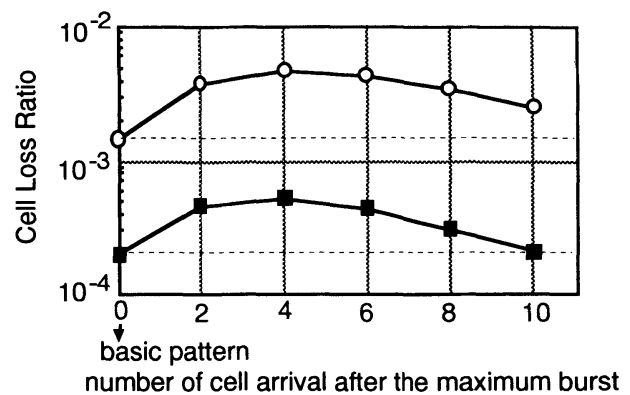

Figure 3 Changes of the CLR and the s.f. values due to the number of cell arrivals after maximum burst. the basic pattern.

In pattern (b) 10 cells arrive at the average rate after the maximum burst, but in pattern (c) 4 cells arrive after the maximum burst. Figure 3 shows the changes of the CLR and the s.f. value with changes in the number of cells arriving after the maximum burst.

We can conclude that the basic pattern (= the conventional "worst pattern") is not always the worst pattern, and that the QOS can not be guaranteed by the BWA method using the basic pattern.

Doshi (1993) has also showed by fluid approximation that the basic pattern is not the worst for a very special case. In his example, the number of multiplexed connections is only two, and the peak arrival rate is the same as the link speed. Our result, however, is for a far more realistic situation and is not based on any approximation.

\section{4 More simulations}

We investigated the relation between pattern and the CLR in detail. In order to reduce the time required for simulation, we changed the LBA parameter and other parameters. The parameters we used this time are as follows: the depth of LB 9.1, the peak arrival rate 0.2 , and the average arrival rate 0.02 . The number of multiplexed cell streams is 48 .

\section{Patterns worse than the basic pattern}

First we compared the CLR of the basic pattern to those of patterns in which some cells arrive at the average rate after the maximum burst (see Figure 4). Again we find the basic pattern is not always the worst. Figure 5 (a) and (b) show that the CLR changes with increases in the number of average rate arrival cells. Figure (a) and figure (b) are for buffer sizes of 75, 100, respectively. For example, the pattern with $\mathrm{N}=4$ in Figure 4 (b) yields 2 times larger CLR 
than the basic pattern (a) in Figure 4. Thus we confirm that the conjecture that "the basic pattern is the worst" does not hold.

We can intuitively understand why patterns like Figure 4 (b) are worse than the basic pattern as follows:

\section{interpretation}

The arrivals of additional cells after maximum burst, see Figure 4 (b), effectively decrease the rate at which the queue length is decreased in the short term after burst collision (i.e., simultaneous arrival of bursts). If burst collisions occur during this short term, cells may be lost.

Sample paths of queue length support this interpretation. Figure 6 (a) shows an example of sample path of queue length and number of lost cells for the basic pattern (a) in Figure 4, and Figure 6 (b) shows that for the pattern with $\mathrm{N}=3$ in Figure 4 (b). In each cell stream, cell arrival begins with the maximum burst at random time, but those starting times are identical for both patterns.

For basic pattern (a), the first burst collision occurs at time $\mathrm{t}=200$ or so, and the second one comes at around $\mathrm{t}$ $=400$. The first burst collision makes the queue length increase rapidly but no cells are lost. The second one arrives after the queue length shrinks enough that no cell loss occurs. For case (b), the first burst collision makes the queue length long enough to cause cell losses. The queue length does not shorten sufficiently before the second burst collision comes, and this causes large cell loss. It is obvious that the

(a) 75 cell buffer

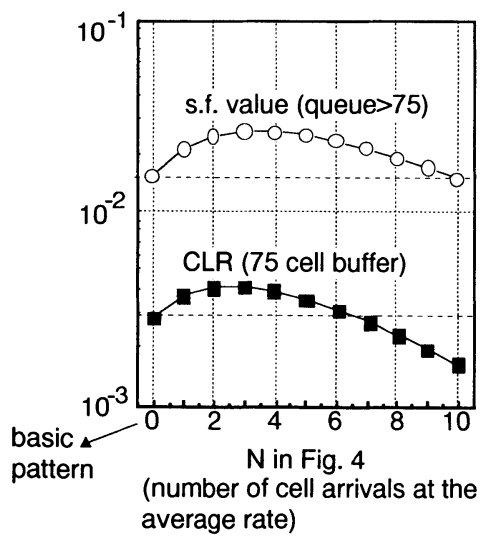

(a) Basic pattern

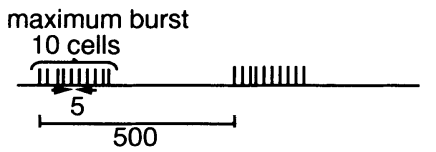

(b) Maximum Burst $+\mathrm{N}$ cells arriving at the average rate $\mathrm{N}$ cell arrivals at the average rate

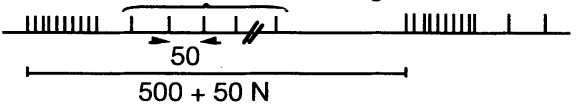

Figure 4 Compared patterns in more simulation.

(b) 100 cell buffer

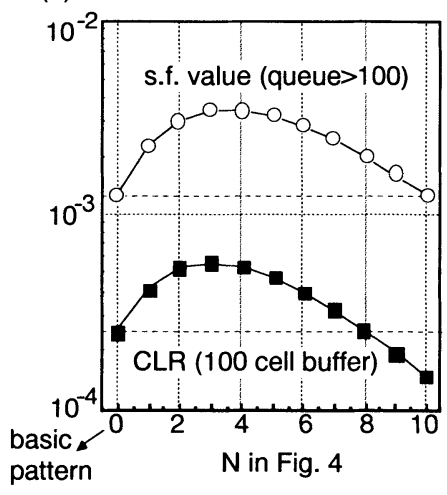

Figure 5 Comparison of CLR and Survivor function value for the pattern in Figure 4. 


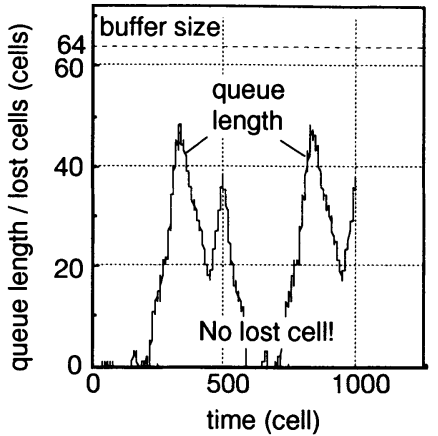

(a) basic pattern (Fig. 4 (a))

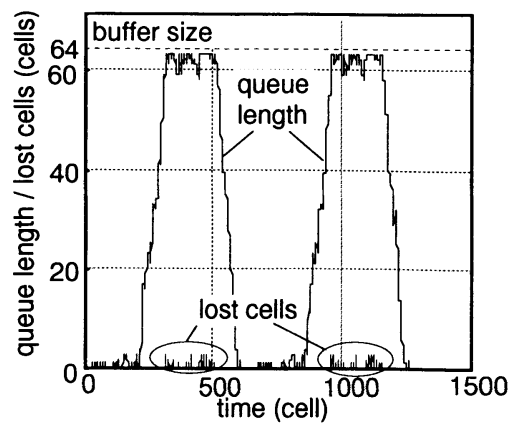

(b) The pattern with $\mathrm{N}=3$ in Fig. 4 (b)

Figure 6 Examples of sample paths of the queue length and the number of lost cells. differences between (a) and (b) result from the average rate cell arrivals after the maximum burst in pattern in Figure 4 (b).

\section{The CLR and the survivor function value}

Figure 5 also shows the relation between the CLR and the survivor function value. Figure 7 shows the relative change of CLR and s.f. values from Figure 5 (b) so that the s.f. value for $\mathrm{N}=0$ is plotted at the same point as the CLR for $\mathrm{N}=0$. For example, in Figure 7, the pattern with $\mathrm{N}=9$ does not yield larger "CLR" than the basic pattern, though the pattern yields larger "s.f. value" than the basic pattern. Thus we can conclude that assumption 1 is not always true, as Worster pointed out.

In the patterns like (c) in Figure 2, the queue length

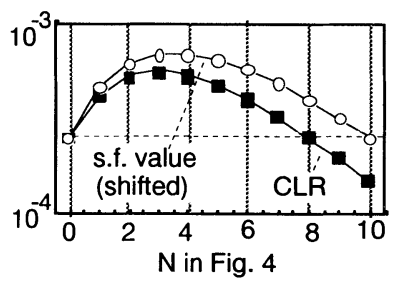

Figure 7 Comparison of CLR and s.f. value in Figure $5(\mathrm{~b})$. decreases more slowly than the basic pattern after the steep increase caused by the burst collision because the average rate cell arrival after the maximum burst (see Figure 6 again). Thus, as Worster mentioned, the survivor function values of the pattern like (c) in Figure 2 can be larger than that of the basic pattern although its CLR remains smaller than the basic pattern.

From Figure 7, however, we can see that the s.f. value is a still good indicator of the relative change in CLR though some differences remain.

\section{The traffic load and the CLR}

As suggested by Yamanaka et al. (1992), we found that some patterns that are worse than the basic pattern at some traffic loads are not worse at lower loads. Figure 8 compares the CLR of the basic pattern and the pattern with 3 average rate cell arrivals $(\mathrm{N}=3$ in Figure 4). At $96 \%$ link usage, the pattern with $\mathrm{N}=3$ shows higher CLR, but the converse is true at smaller loads.

This result implies that as the load lightens, the effect of the additional cell arrivals after maximum burst on the behavior of the queue length becomes small. In the light load case, the probability that the second burst collision occurs close to the first burst collision is small, so the additional cell arrivals after maximum burst does not effect the CLR so much. 
(a) buffer size: 75 cells

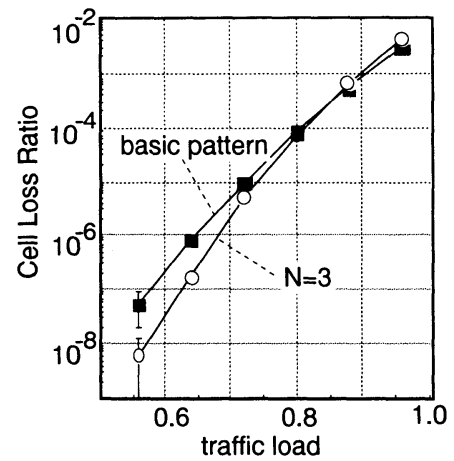

(b) buffer size: 100 cells

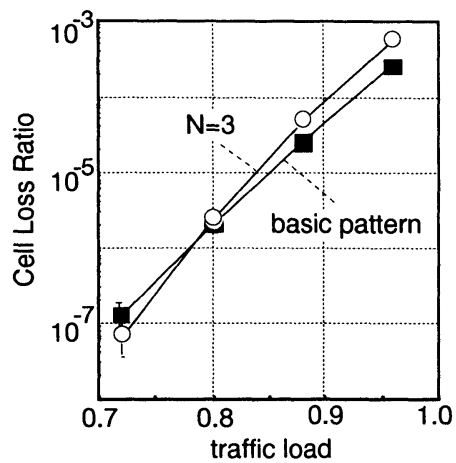

Figure 8 Change of CLR due to traffic load reduction.

\section{Other various patterns and the CLR}

Next we investigated other various patterns. For explanation, we define "Child Pattern" (Figure 9) following Yamanaka et al. (1992). A Child Pattern is defined by the number of cells in it, and we call this number the "Child Pattern Cell Number (CPCN)". The length of a Child Pattern is the average cell arrival interval multiplied by CPCN. In the Child Pattern defined here, as shown in Figure 9, all cells arrive at the peak rate in the end of the pattern.

We performed a simulation for patterns like in Figure 10. This pattern is generated by inserting into the basic pattern several identical Child Patterns after the maximum burst. We call the number of times the Child Pattern is repeated the "Child Pattern Iteration Number (CPIN)". For pattern (b) in Figure 2, CPCN $=1$ and CPIN $=10$. Changing CPCN and CPIN produces various periodic cell patterns. Notice that these variations yield identical LBA parameters, i.e., the average arrival rate, the peak arrival rate, and the depth of the LB, so they are passed by the LBA that passes the basic pattern.

Figure 11 shows the CLRs for patterns with various $\mathrm{CPCN}$ and CPIN values. Each curve in Figure 11 shows CLRs for patterns with constant CPCN but different CPINs. The increase in CPIN increases the period of the pattern as a whole, so we let the horizontal axis of Figure 11 depict the pattern period. We can see the following things: when CPCN is small, the CLR first increases and then decreases as CPIN increases. When CPCN exceeds 3, the CLR monotoni-

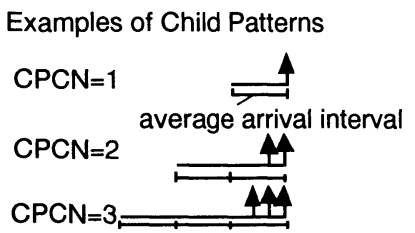

Figure 9 Chile Patterns.

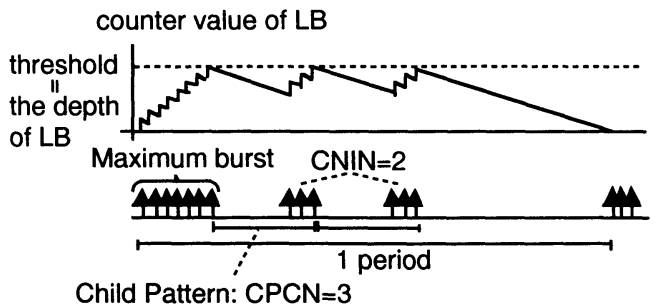

Child Pattern: $\mathrm{CPCN}=3$

Figure 10 A new pattern generated by iterated Child Pattern. 
cally decreases.

Many iterations of the Child Pattern greatly increase the whole pattern period, which makes the probability of burst collision small. Thus, if CPIN is large enough, the CLR is less than that of the basic pattern. Large CPCN increases the distance between the maximum burst and the Child Pattern, thus the Child Patterns have less effect on the queue length after burst collision, and thus make CLR less than the basic pattern. Only the patterns whose CPCN and CPIN are both small can cause larger CLRs than the basic pattern.

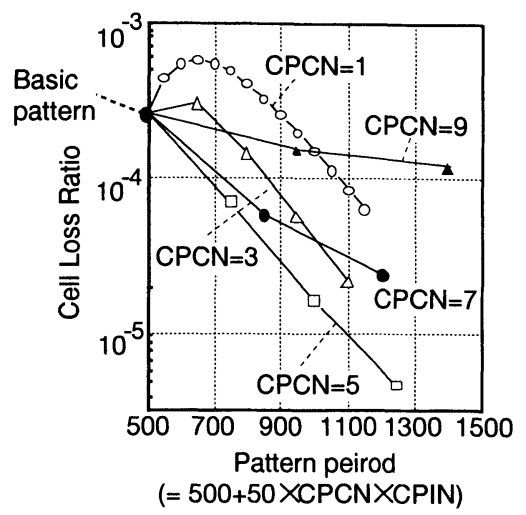

\begin{tabular}{|ll|}
\hline Number of cells in the Maximum burst & 10 \\
Depth of the LB & 9.1 \\
Peak Arrival Rate & 0.2 \\
Number of multiplexed cell streams & 48 \\
Buffer size & 100 \\
\hline
\end{tabular}

Figure 11 CLR for various CPCN and CPIN.

\section{HOW THE RELATION BETWEEN CLR AND PERIODIC PATTERN OF CELL ARRIVAL DEPENDS UPON LBA PARAMETERS}

In the previous section, we investigated in detail the relation between CLR and periodic cell patterns for a specified set of LBA parameters. In this section, using an approximation method for the survivor function we examine how the relation between CLR and periodic cell patterns depends upon LBA parameters, i.e., the average arrival rate, the peak arrival rate and the depth of the LB.

Since we confirm that the basic pattern is not always the worst, we should estimate the impact of that fact on Connection Admission Control. Thus we focus on the maximum permissible link load at a specified CLR. For many LBA parameters, we compare the maximum permissible link load for the basic pattern with that of other patterns, calculating them with an approximation method for s.f. values, which is proposed in this section.

\section{1 approximation method for survivor function value}

We use the following model: assume that time is discrete and define the transmission time of a cell as the unit of time. Departures of cells take place at the beginning of slots, and arrival during slots. The buffer size is infinite.

Let $Q$ be the queue length. Define $a_{t}$ as the number of arriving cells at time $t$ (slots) and $Q(t)$ as the queue length at the end of the slot. The following lemma is valid for the case of periodic cell streams (Bhargava, 1989). This is a special application of Benes results for a G/ $\mathrm{G} / 1$ stochastic process (Benes, 1963) to the deterministic process. 


\section{Lemma 1}

Assume $Q\left(t_{0}\right)=0$ at time. When periodic cell streams with period $T$ are multiplexed and $t$ is greater than $t_{0}+T$, the survivor function is given as:

$$
\operatorname{Pr}[Q(t)>q]=\sum_{w=1}^{T} \operatorname{Pr}\left[A_{w} \cap B_{w}\right]=\sum_{w=1}^{T} \operatorname{Pr}\left[A_{w}\right] \operatorname{Pr}\left[B_{w} \mid A_{w}\right]
$$

where

$$
A_{w}=\left\{\sum_{i=0}^{w-1} a_{t-i}-w=q\right\}, \quad B_{w}=\left\{\text { for }{ }^{\forall} j(w<j \leq T), \sum_{i=0}^{j-1} a_{t-i}-j<q\right\} .
$$

(proof) Refer to Bhargava et al. (1989).

For VBR (Variable Bit Rate) cell streams, $\operatorname{Pr}\left[B_{w} \mid A_{w}\right]$ is difficult to calculate, but we can obtain upperbound as follows (Norros, 1991) (Sato, Y., 1992) :

\section{Proposition 1}

The upperbound of the survivor function is given as:

$$
\operatorname{Pr}[Q(t)>q] \leq \sum_{w=1}^{T} \operatorname{Pr}\left[A_{w}\right]=\sum_{w=1}^{T} \operatorname{Pr}\left[X_{w}=W+q\right]
$$

where $X_{w}$ is the number of cells during $w$ slots.

Assume that $n$ periodic cell streams are multiplexed and that their phases are mutually independent. The calculation of the term $\sum_{w=1}^{T} \operatorname{Pr}\left[X_{w}=W+q\right]$ of equation (1) is time consuming because it requires a number of $\mathrm{n}$-fold convolution of probability distributions.

We apply the "shifted normal approximation" (Norros, 1991) to avoid convolution. For homogeneous traffic, where all cell streams have the same periodic pattern, we can approximate each term of the summation in the right hand of equation (1) as follows:

\section{Proposition 2}

Assume that $n$ cell streams with the same periodic pattern are multiplexed. Let $X_{k, w}$ be the number of cells arriving during $w$ slots for the $k$ th cell stream. is identical for all $(1 \leq k \leq n)$ so we define

$$
P_{w}(x) \equiv \operatorname{Pr}\left[X_{k, w}=x\right]
$$

Then,

where

$$
\operatorname{Pr}\left[X_{w}=w+q\right]=\exp \left[n \mu_{w}\left(s_{w}\right)-s_{w} \mu_{w}^{\prime}\left(s_{w}\right)\right] \times\left\{\frac{1}{\sqrt{2 n \mu_{w}^{\prime \prime}\left(s_{w}\right)}}+o\left(\frac{1}{\sqrt{n}}\right)\right\},
$$




$$
g_{w}(s) \equiv \sum_{x} \exp (s x) P_{w}(x)
$$

$g_{w}(s)$ is the moment generating function. $s_{w}$ is the value of $s$ such that $n \mu_{w}^{\prime}\left(s_{w}\right)=w+q$. (proof) Refer to Chapter5, Appendix 5A in Gallager (1968).

Using the Chernoff bound, Nakagawa (1993) has shown a looser upperbound of the right hand of equation.

\section{2 Evaluation of the precision of the approximation method}

Figures 12 to 15 compare the approximated survivor function values with the true survivor function values and CLR, which we obtained by the simulation in section 3 . Figure 12 shows an approximation of the simulation values given in Figure 8 (a). Figure 14 compares approximation values for $\mathrm{CPCN}=1$ and $\mathrm{CPCN}=5$ with the values given in Figure 11. Comparing Figure 12 and Figure 14, the upperbound for survivor function value is at most 1 order larger than true s.f. value and 2 orders larger than the CLR. The relative changes, however, seem well approximated.

Figure 13 compares the relative changes in the simulation values and approximation values given in Figure 12. We plot each curve so that they meet at the load of 0.8 . We can see that if we consider only the neighborhood of the 0.8 load, the relative changes of s.f. value and CLR are well approximated by the upperbound approximation. Thus the s.f. approximation yields the relative difference in the permissible maximum load for a given CLR between the basic pattern and other patterns.

Figure 15 compares the relative changes in simulation values and approximation values given in Figure 14. There still remains a slight discrepancy between the upperbound for the s.f. and the true s.f. value or the CLR. The approximation, however, well traces the pattern of changes.

(a) basic pattern

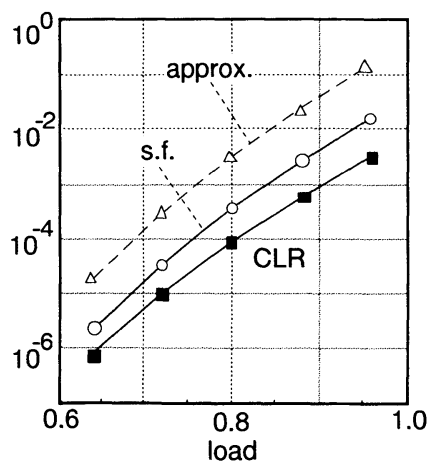

(b) the pattern with $\mathrm{CPCN}=1, \mathrm{CPIN}=3$

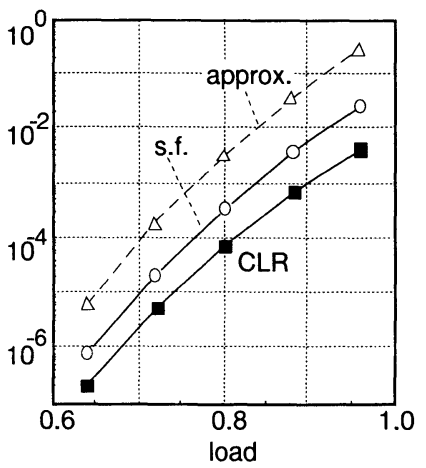

\# buffersize: 75 cells

\# survivor function value: $\operatorname{Pr}[q u e u e>75]$

Figure 12 Comparison of CLR, s.f. value, and upperbound approximation for s.f. value. 
(a) basic pattern

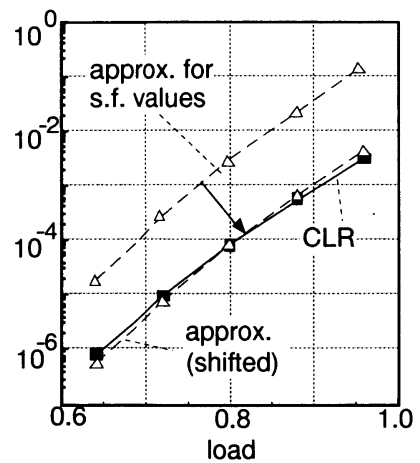

(b) the pattern with $\mathrm{CPCN}=1, \mathrm{CPIN}=3$

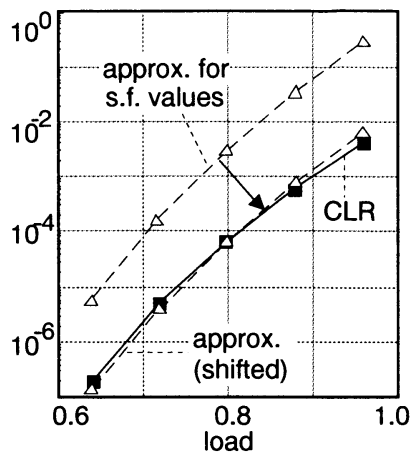

\# buffersize: 75 cells

\# survivor function value: $\operatorname{Pr}[q u e u e>75$ ]

Figure 13 Comparison of change of CLR and approximated s.f. value with the load change.

(a) $\mathrm{CPCN}=1$

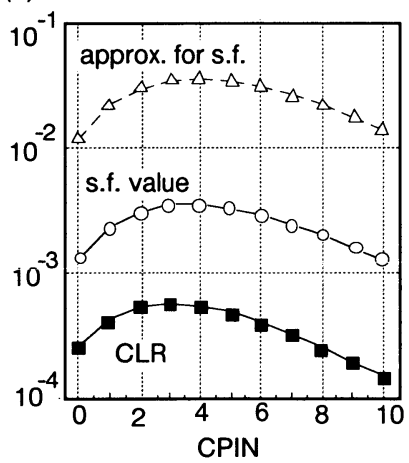

(b) $\mathrm{CPCN}=5$

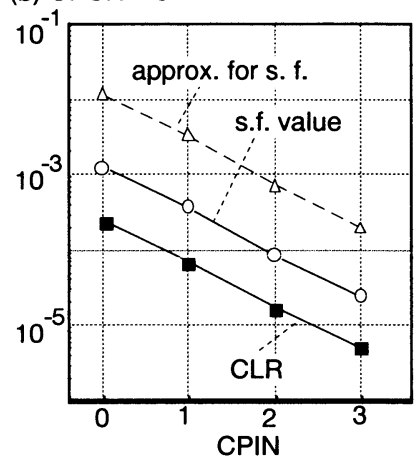

\# buffersize: 100 cells, survivor function value: $\operatorname{Pr}[q u e u e>100]$

Figure 14 Comparison of CLR, s.f. value and approximated s.f. value.

(a) $\mathrm{CPCN}=1$

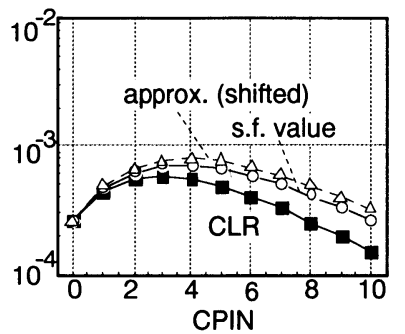

(b) $\mathrm{CPCN}=5$

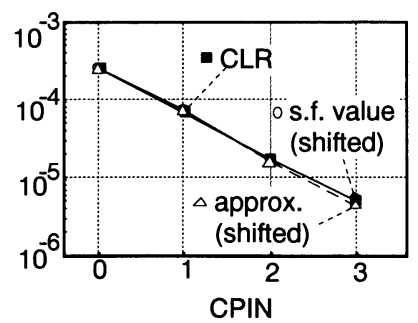

Figure 15 Comparison of change of CLR, s.f. value and approximated s.f. value with change in CPIN. 


\section{3 Dependence of the relation between CLR and periodic pattern of cell arrival upon LBA parameters}

In this section, using the above upper bound approximation, we examine how the relation between CLR and periodic cell pattern depends upon various LBA parameters, i.e., the average arrival rate, the peak arrival rate, and the depth of the LB.

Since there are infinitely many periodic patterns for each set of LBA parameters, it is impossible to investigate all of them, so we examined only the periodic patterns described in section 3.4. Any periodic pattern consists of bursts of various scale, and the periodic patterns described in section 3.4 are combinations of large and small bursts. Thus we believe we can obtain an overview of the relation between CLR and periodic pattern.

It is best to compare the CLR of the basic pattern with those of the other patterns for wide range of link loads and LBA parameters. In order to reduce the calculation time and to look at the data from the CAC point of view, however, we compare the maximum link load of each pattern given a specific CLR. We calculate the maximum link load at which the approximated s.f. value is just less than the given CLR. The value of the maximum link load itself is not so precise because approximated s.f. values are 1 order or more larger than the CLR, but we can well approximate the difference in loads at that load level.

\section{LBA parameters}

We examined the LBA parameters as comprehensively as possible. We investigated 44 combinations of the following parameters:

- Average arrival rate $=\{0.01,0.05,0.002\}$

- Peak arrival rate $=\{1,0.2,0.1,0.002\}$

- Number of cells in themaximum burst ( $\cong$ the Depth of the LB) $=\{10,20,50,100\}$

- $\mathrm{CLR}=10^{-10}$

- Buffer size $=256$

The average arrival rate of 0.01 means that, for example, the average rate of the connection is $1.5 \mathrm{Mbps}$ if the capacity of the transmission link is $150 \mathrm{Mbps}$.

As in section 3.4, we examined the following patterns: the CPCN ranges from 1 to one half the number of cells in maximum burst. The CPIN is increased until the approximated s.f. values begins to decrease. In order to reduce the computation time, we examined only values of $\{1,2,5,10,20,50,100\}$ for both CPCN and CPIN.

\section{Dependence on $L B A$ parameters}

For 9 sets of the 44 sets examined, there exist some patterns that yielded approximated s.f. values larger than the basic pattern. We call these 9 sets of LBA parameters the dangerous sets hereafter. Among the dangerous sets, the reduction in the maximum load between the identified worst pattern and the basic pattern is

$$
\begin{array}{ll}
\text { maximum } & 0.03 \\
\text { average } & 0.015
\end{array}
$$

(These are not relative reduction rate, but absolute reduced values of loads).

Figure 16 shows the reduction in the maximum load permitted by the identified worst pattern against that of the basic pattern. We can see the following from Figure 16; 


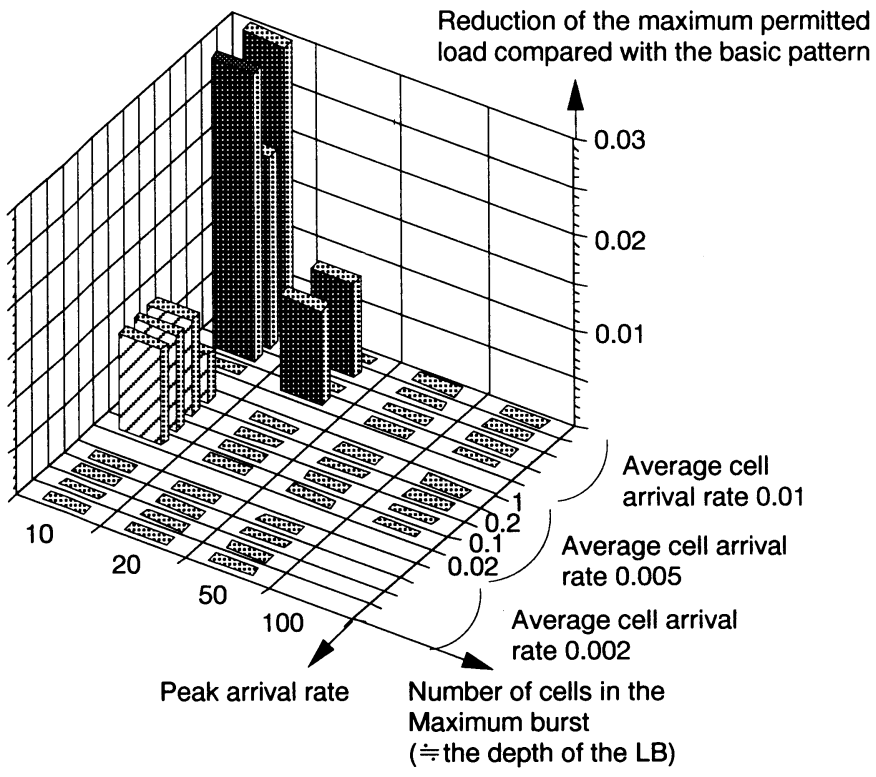

Figure 16 Reduction of the permissible maximum load of the identified worst patterns from that of the basic patterns (calculated by using approximation for $\mathrm{s}$. f. value).

1. In all the dangerous sets, the number of cells in the maximum burst ( $\cong$ the depth of the LB) is small (10 or 20$)$.

2. As the average arrival rate decreases, the dangerous sets become fewer, and the reduction in maximum load becomes smaller.

We also found the below facts:

3. No pattern with CPCN or CPIN greater than or equal to 5 yielded smaller maximum loads (i.e., larger s.f. values) than the basic pattern.

Using the approximation, we examined how the relation between s.f. values and periodic pattern depends upon the link load.

4. The pattern that causes a larger s.f. value than the basic pattern at some load actually yields a smaller s.f. value than the basic pattern when the value of the load decreases.

Fact 3 supports the observation made in section 3.4. Fact 1 and fact 2 are interesting results. We can explain fact 1 and 2 from fact 4 though we do not yet mathematically understand the causes of these phenomena.

If we increase the number of cells in the maximum burst (the depth of the LB) and keep the 
other parameters unchanged, the cell stream becomes more bursty. Thus we have to decrease the load in order to keep the CLR unchanged, so the basic pattern becomes the worst by the logic implied by fact 4 . This may be the cause of fact 1 .

If we make the average arrival rate smaller and keep the other parameters unchanged, the cell stream becomes more bursty because the difference between the average arrival rate and the peak arrival rate becomes larger. Again, we have to decrease the load in order to keep the CLR unchanged. This may be the cause of fact 2 .

\section{BANDWIDTH ALLOCATION METHOD}

As seen from the above results, the conventional bandwidth allocation method that uses the basic pattern should not be applied as it is. Though more precise analysis is needed, we can expect that a rather small safety margin, i.e., $5 \%$ or so for the parameters we examined, for the maximum load is enough to guarantee the negotiated CLR.

Thus, in private networks (including LAN and MAN), the conventional BWA using the basic pattern is good enough to estimate the required bandwidth, because the required QoS and resource utilization efficiency are not so strict or severe in such an environment.

In the public network, the operator who is to offer CBR/VBR ATM connections with strictly guaranteed QOS needs a more precise analysis to decide the necessary margin for the maximum load. For a CBR connection, to adopt a shaper is a good alternative in public networks. The shaper provides the operator with a simple BWA method $(\mathrm{M} / \mathrm{D} / 1$ approximation (Uematsu, 1990) ) and high link utilization of the link (ex. 95\% for 256 cell buffer and CLR of $\left.10^{-9}\right)$.

There is another BWA method (Saito, 1992) that uses only average arrival cell number and maximum arrival number in a fixed size window and has been proven mathematically to guarantee the CLR. It's drawback is that the maximum permissible load is too small for some sets of the LBA parameters. Figure 17 compares the maximum load yielded by Saito's method with that estimated from the identified worst pattern. For VBR connections whose maximum burst length is large, Saito's method gives rather ideal loads, and the method may be satisfactory.

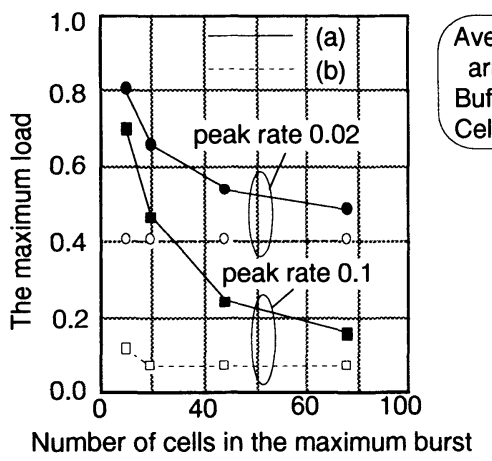

(a) values by the method in section 4.1

(b) values by the method of Saito (1992)

Figure 17 Comparison of the maximum load by the method of Saito (1992) and the load by the method of section 4.1. 


\section{CONCLUSION}

Considering the multiplexing of periodic cell streams allowed by the LB (Leaky Bucket) algorithm, the relation between Cell Loss Ratio (CLR) and periodic cell pattern was investigated.

First, we showed by simulation that the conventional "worst" periodic pattern is not the worst, i.e., other periodic patterns can cause larger CLRs. Therefore the conventional BWA method that uses the basic pattern may not guarantee the negotiated CLR as it is. Second, we investigated in detail, by simulation, how the CLR depends on the periodic pattern. Third, we examined how the relation between CLR and periodic pattern is modified by the LBA parameters. To this end, we used an approximation for the survivor function value of the queue length of periodic cell streams. The approximation applies Benes's G/G/1 result and the "shifted normal approximation". We found that the sets of LBA parameters wherein the basic pattern does not yield the worst performance is limited to those sets with small numbers of cells in the maximum burst. We also found that small safety margins for the maximum permissible load, i.e., $5 \%$ or so for the parameters we examined, are sufficient to guarantee the CLR, if we use the basic patterns to calculate the necessary bandwidth.

In order to establish the ideal BWA method that can strictly guarantee the QOS and maximize the utilization of transmission links, a more accurate analysis is needed for cell streams passing though the LBA-based UPC.

\section{APPENDIX A}

For the computer simulation of section 3, we obtained the CLR for each pattern as follows:

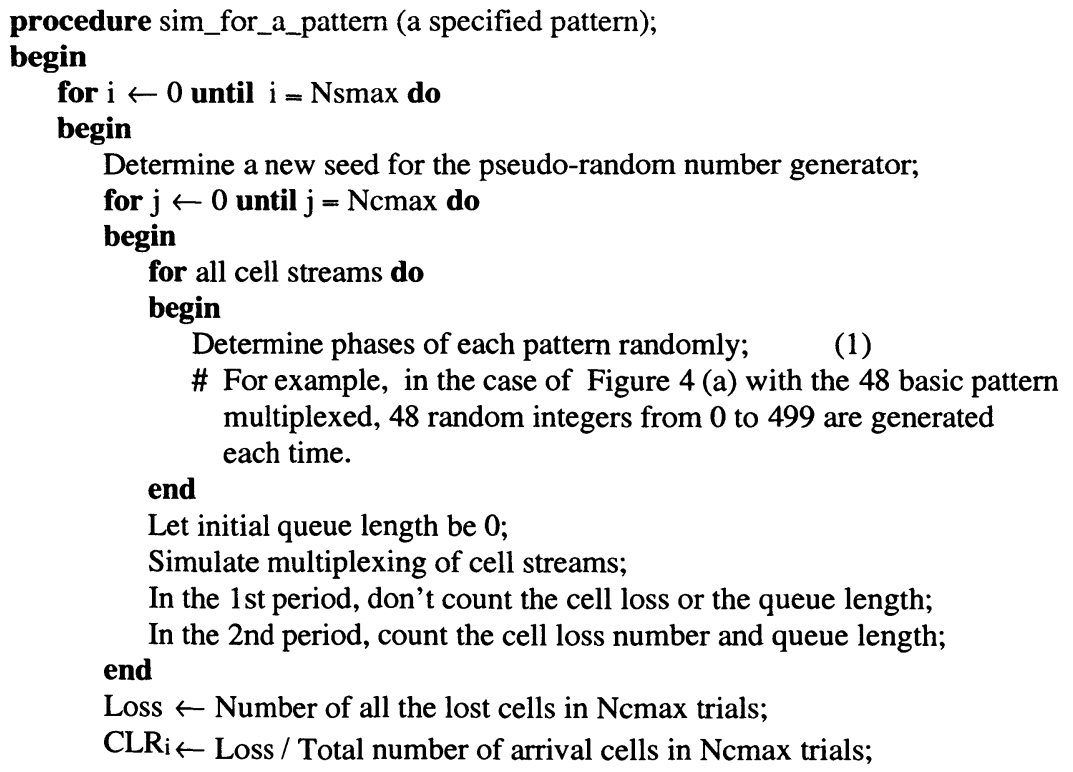




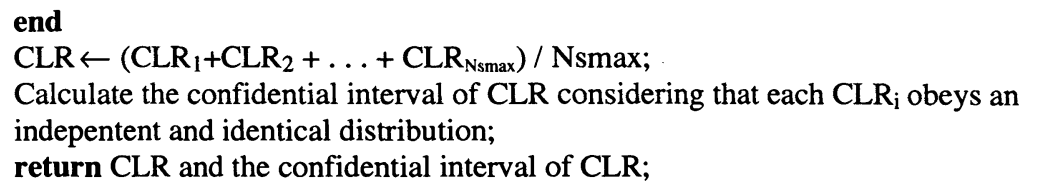

In Figure 4 (a) case with the 48 basic pattern multiplexed Nsmax is 50, Ncmax is 100000 and one period is 500 cell times. So the seed is changed 50 times and the total observed simulation time is $50 \times 100000 \times 500=2.5 \times 10^{9}$ cell times.

The pseudo-random generator is required, for example in the case of Figure 4 (a), to be 48-distributed (see Appendix B) at step (1).

\section{APPENDIX B}

Our simulation requires that the pseudorandom number generator should yield, for example, a 48 dimension vector uniformly distributed in the unit hyper-cube with precision up to $10^{-3}$, i.e., a pseudorandom vector consisting of 48 sequences $\left(x_{n+1}, x_{n+2}, \cdots, x_{n+48}\right)$ from the generator should be really randomly distributed in $48 \mathrm{D}$ cube (see Appendix A). Though a pseudo-random number generator should be a "real" random number generator, this condition is naturally achieved, we can use only a pseudo-random number generator, which generates numbers deterministically. This multidimensional equidistribution property is called the $\mathrm{k}$ distribution property (Knuth, 1981).

The popular Linear Congruential generator with, for example, a 248 period can guarantee at most only 8-distribution (8D equidistribution) with precision about $0.016\left(=2^{-6}\right)$. Thus we used a Generalized Feedback Shift Register generator with the initializing method by Fushimi and Tezuka (Fushimi, 1983). We used the prime trinomial $x^{p}+x^{q}=1$ with $\mathrm{p}=512, \mathrm{q}=32$ and generate 32 bits binary fractions. The period of the sequence is 2512 . For example, this generator achieves 10-distribution with precision of 2-32, and 49-distribution with precision of 2-10 (Fushimi, 1989, chap1, sec 2. 2).

\section{ACKNOWLEDGMENT}

The authors wish to thank Dr. Haruo Yamaguchi, Dr. Hiromi Ueda and Dr. Naoaki Yamanaka of NTT Network Service Systems Laboratories and Dr. Hiroshi Saito of NTT Telecommunication Network Laboratories for their helpful advice.

\section{REFERENCE}

ATM forum UNI spec. ver. 3.0 (1993).

Benes, V.E. (1963), General Stochastic Processes in the Theory of Queues, Addison Wesley. Bhargava, A. et al. (1989) Queuing analysis of continuous bit stream transport in packet networks, in Proc. IEEE GLOBECOM' 89, pap. 25. 6, 903-7. 
Doshi, B. T. (1993) Deterministic Rule Based Traffic Descriptors for Broadband ISDN: Worst Case Behavior and Connection Admission Acceptance Control, Proc. IEEE GLOBECOM'93, pap. 48A. 1, 1759-64.

Fushimi, M. and Tezuka, S. (1983) The k-distribution of Generalized Feedback Shift Register Pseudorandom Generator, Commun. ACM, 26, 516-23.

Fushimi, M. (1989) Ransuu (Pseudo-Random Number) (in Japanese), Univ. of Tokyo press, Tokyo.

Gallager, R.G. (1968) Information Theory and Reliable Communication, John Wiley \& Sons, N. Y.

ITU-T Recommendation I. 371 (1993) Traffic Control and Congestion Control in B-ISDN, Geneva .

Kositpaiboon, R. and Phung, V. (1990) Usage Parameter Control and Bandwidth Allocation for B-ISDN/ATM Variable Bit Rate Services, Multimedia ' 90 Session 4, Bordeaux, France.

Knuth, D.E. (1981) The Art of Computer Programming, Vol. 2: Seminumerical Algorithm, 2nd ed., Addison-Wesley, Reading, MA.

Nakagawa, K. (1993) Loss and Waiting Time Probability Approximation for General Queuing, IEICE Tech. Rep., SSE92-2.

Niestegge, G. (1990) The 'leaky bucket' policing method in the ATM (Asynchronous Transfer Mode) Network, Int. J. Digital and Analog Commun. Sys., 5, 203-15,.

Norros, I. et al. (1991) The superposition of Variable Bit Rate Source in an ATM Multiplexer, IEEE J. SAC., 9, no. 3, 378-87.

Saito, H. (1992) Call Admission Control in an ATM Network Using Upper Bound of Cell Loss Probability, IEEE Trans. Commun., 40, no. 9, 1512-21.

Sato, K. et al. (1990) Broad-Band ATM Network Architecture Based on Virtual Path, IEEE Trans. Commun. 38, no. 8, 1212-22.

Uematsu, H (1990) IEICE Tech. Rep., CS90-43.

Sato, Y. et al. (1992) Analysis of Multimedia VBR Traffic Multiplexing Characteristics (in Japanese), IEICE National Spring Conf. , B-695.

Yamanaka, N. et al. (1992) Precise UPC scheme and bandwidth allocation methods for ATMbased BISDN characterized by wide-ranging traffic parameter values, Int. J. Digital and Analog Commun. Sys., 5, 203-15.

Worster, T. (1994) Modeling Deterministic Queue: The Leaky Bucket as an Arriving Process, ITC 14.

\section{BIOGRAPHY}

Kei Yamashita was born in Hokkaido, Japan, in October 1967. He received the B.S. and M.S. degrees in Mathematical Engineering from the University of Tokyo, Japan in 1990 and 1992. He joined Nippon Telegraph and Telephone Corporation (NTT) in 1992 and is currently working for the NTT Optical Network Systems Laboratories. He has been engaged in research and development of traffic control techniques in ATM networks.

Mr. Yamashita is a member of the Institute of Electronics, Information, and Communication Engineers (IEICE) of Japan. 
Youichi Sato was born in Nagano, Japan, in August 1963. He received the B.S. degrees in electric engineering from the Science University of Tokyo in 1986. He joined the Electrical Communication Laboratories of Nippon Telegraph and Telephone Corporation in 1986. He is currently a Research Engineer in NTT Transmission Systems Laboratories. His current research interests include performance analysis, bandwidth allocation strategies and cell traffic control techniques in ATM networks.

Mr. Sato is a member of the Institute of Electronics, Information, and Communication Engineers (IEICE) of Japan and of the IEEE Communications Society. 